\title{
MCP-PMT Studies at the High-B Test Facility at Jefferson Lab
}

\author{
Y. Ilieva ${ }^{a *}$, L. Allison ${ }^{b}$, T. Cao ${ }^{a}$, G. Kalicy ${ }^{b}$, P. Nadel-Turonskic ${ }^{c}$, K. Park ${ }^{b}$, C. Schwarz ${ }^{d}$, \\ J. Schwiening ${ }^{d}$, and C. Zorn ${ }^{c}$ \\ ${ }^{a}$ University of South Carolina, \\ 712 Main St., Columbia, SC 29208, USA \\ E-mail: ilievadsc.edu \\ ${ }^{b}$ Old Dominion University, \\ 4600 Elkhorn Ave, Norfolk, VA 23529, USA \\ ${ }^{c}$ Thomas Jefferson National Accelerator Facility, \\ 12000 Jefferson Avenue, Newport News, VA 23606, USA, \\ ${ }^{d}$ GSI Helmholtzzentrum für Schwerionenforschung GmbH, \\ Planckstraße 1, 64291 Darmstadt, Germany
}

\begin{abstract}
Here we present preliminary results for the gain performance of commercially available 3- $\mu \mathrm{m}$ and 6- $\mu \mathrm{m}$ pore-size single-anode microchannel-plate photomultipliers (MCP PMTs) in magnetic fields up to $5 \mathrm{~T}$ and for various orientations of the sensor relative to the field direction. The measurements were performed at Thomas Jefferson National Accelerator Facility in Newport News, VA. Our results show that smaller-pore-size PMTs have better gain performance in magnetic fields. At various angles, the shape of the gain dependence on the strength of the magnetic field strongly depends on the type of the sensor. Also, for each sensor, the azimuthal dependence is strongly correlated with the polar angle. Overall, the sensors exhibit a reasonable performance up to $2 \mathrm{~T}$, although that upper limit depends on the sensor, the applied high voltage, and the orientation of the sensor relative to the field. To optimize the operational and design parameters of MCP PMTs for performance in high magnetic fields, further measurements and simulation studies will be pursued. Our studies are part of an R\&D for development of a Detector of Internally Reflected Cherenkov Light for the central detector of a future U.S. Electron Ion Collider.
\end{abstract}

KEYWORDS: MCP PMTs, Cherenkov detectors, Photon detectors for UV, visible and IR photons (solid-state).

${ }^{*}$ Corresponding author 


\section{Contents}

1. Introduction 目

2. Experimental Setup 2]

3. Results and Discussion

田. Summary 8

\section{Introduction}

A polarized Electron Ion Collider (EIC) is the next large U.S. facility for nuclear physics research. Some of the main scientific objectives of the facility will be to map the gluon and sea quark distributions in space and in momentum space, to study gluon and sea quark polarizations, nuclear quark and gluon distributions, and parton propagation in nuclei [1]]. Compared to previously operated colliders, the EIC will feature a polarized electron beam (eventually, the proton and the light-ion beams could also be polarized), variable center-of-mass (CM) energy, and a high luminosity $\left(10^{33-34} \mathrm{~cm}^{-2} \mathrm{~s}^{-1}\right.$ for ep collisions, for example).

The EIC was first recognized as an important future for U.S. nuclear physics in the 2007 Long Range Plan recommended to the Department of Energy (DOE) by the Nuclear Science Advisory Committee (NSAC) [2]. In this plan, the NSAC recommended that resources be allocated for the development of accelerator and detector technology to lay the foundation for a polarized EIC. In the 2015 Long Range Plan, a high-energy, high-luminosity, polarized EIC was classified as the highest priority for new facility construction and recommended for funding [3]. Since the earlier allocation of R\&D EIC funding, efforts at Brookhaven National Laboratory (BNL) and at Thomas Jefferson National Accelerator Facility (Jefferson Lab) have been ongoing to develop competing designs of the EIC and its detector systems at their sites. While the location of the EIC is not yet finalized, R\&D projects at the two labs and at U.S. universities have been investigating the limits of related detector and accelerator technology.

The Jefferson-Lab EIC design plans for two interaction points (IP) initially. The detector design for one of the interaction points (IP1) is of a barrel magnetic spectrometer equipped with tracking and particle identification (PID) detectors (see Fig. (1). One of the PID detectors in the barrel is a Detection of Internally Reflected Cherenkov light (DIRC) device. The studies presented here started as part of the specific DIRC development R\&D, although our results also benefit the other EIC PID detectors. The limited space available for DIRC integration and the larger momenta of the outgoing particles are unique characteristics that an EIC DIRC design must address [ [4]. For example, a pion/kaon separation above $4 \mathrm{GeV} / c$ needs to be achieved. These characteristics place 


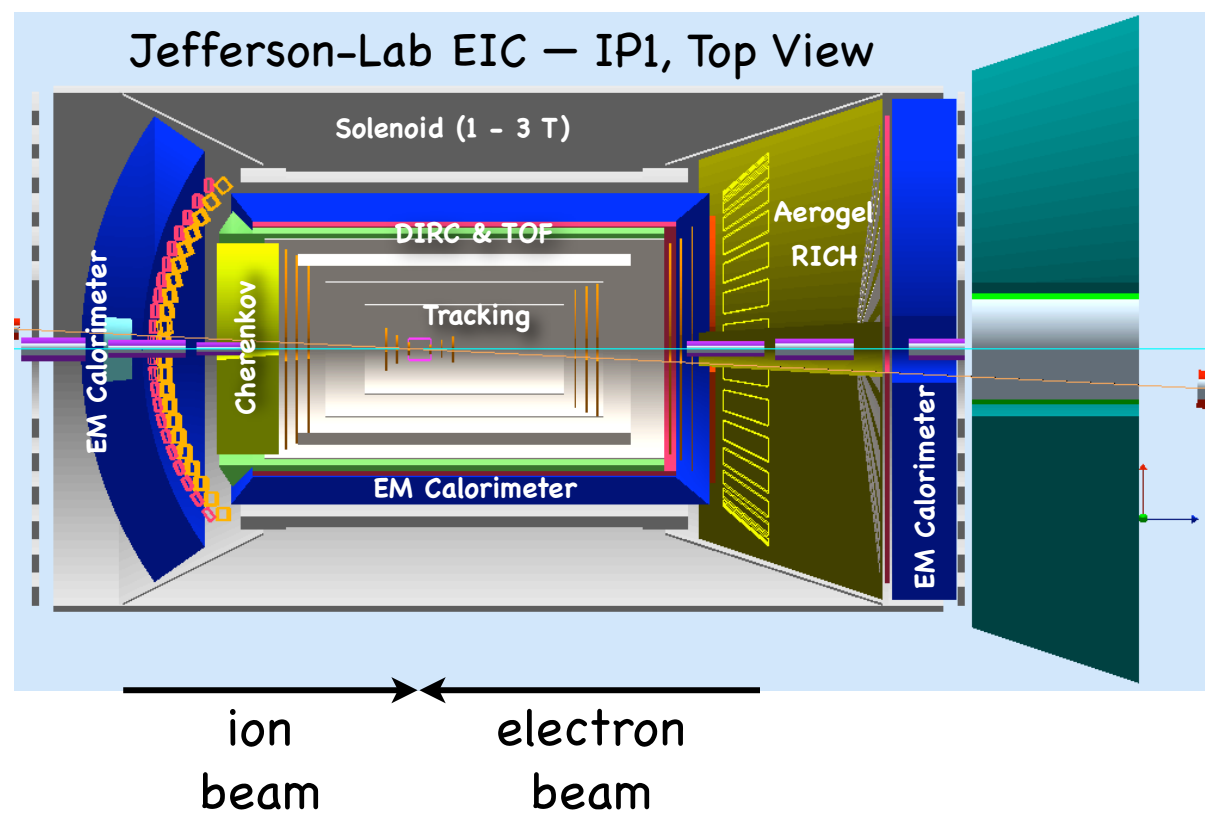

Figure 1. Top view of the detector design for IP1 of the Jefferson-Lab EIC solution. The magnetic field is provided by a dual solenoid. PID detectors include electro-magnetic calorimeters, a fast time-of-flight detector, and several Cherenkov detectors, such as an aerogel, or modular, Ring Imaging Cherenkov (RICH) and a DIRC. Due to space limitations the DIRC readout must be very compact and may need to be operated in the fringe field of the solenoid, i.e. in a non-uniform magnetic field of a magnitude of $3 \mathrm{~T}$ or higher. The figure is a courtesy of Zhiwen Zhao.

unique requirements on the DIRC readout sensors, which must have small pixel size, to accommodate the compact size of the expansion volume and satisfy the DIRC resolution requirements, and have high tolerance to magnetic fields as they may need to be operating in a non-uniform magnetic field of magnitude of about $3 \mathrm{~T}$ (and possibly higher). The studies of gain performance of smallpore-size microchannel-plate photomultipliers (MCP PMTs) in magnetic fields up to $5 \mathrm{~T}$ presented here, aim to identify the operational limits of commercially available sensors and, eventually, to support the optimization of MCP-PMT design and operational parameters for implementation in the EIC DIRC, as MCP PMTs offer good timing and a large number of pixels over a small area.

Previously, MCP-PMT performance in magnetic fields has been studied experimentally [5], [6, [7, 8, 9, [10] in fields up to $2 \mathrm{~T}$. Theoretical calculations have been only done for axial magnetic fields [11], [12]. Our measurements are the first in fields above $2 \mathrm{~T}$.

\section{Experimental Setup}

The results we present here are based on data taken at Jefferson Lab. The setup makes use of a superconducting solenoid magnet with a cylindrical warm bore of a diameter of $12.7 \mathrm{~cm}$ and a length of $76.2 \mathrm{~cm}[13$, [14]. The magnet generates a central field with inhomogeneity of less than $5 \times 10^{-5}$ over a cylindrical volume of a diameter of $1.5 \mathrm{~cm}$ and length of $5 \mathrm{~cm}$, which is adequate for the size of the tested sensors. The magnitude of the field is adjustable and can reach up to $5 \mathrm{~T}$. The sensor being tested is placed in a custom built non-magnetic, light-tight cylindrical dark box 
(see the left panel of Fig. [ for a photograph of the setup). Inside the dark box, the sensor is placed
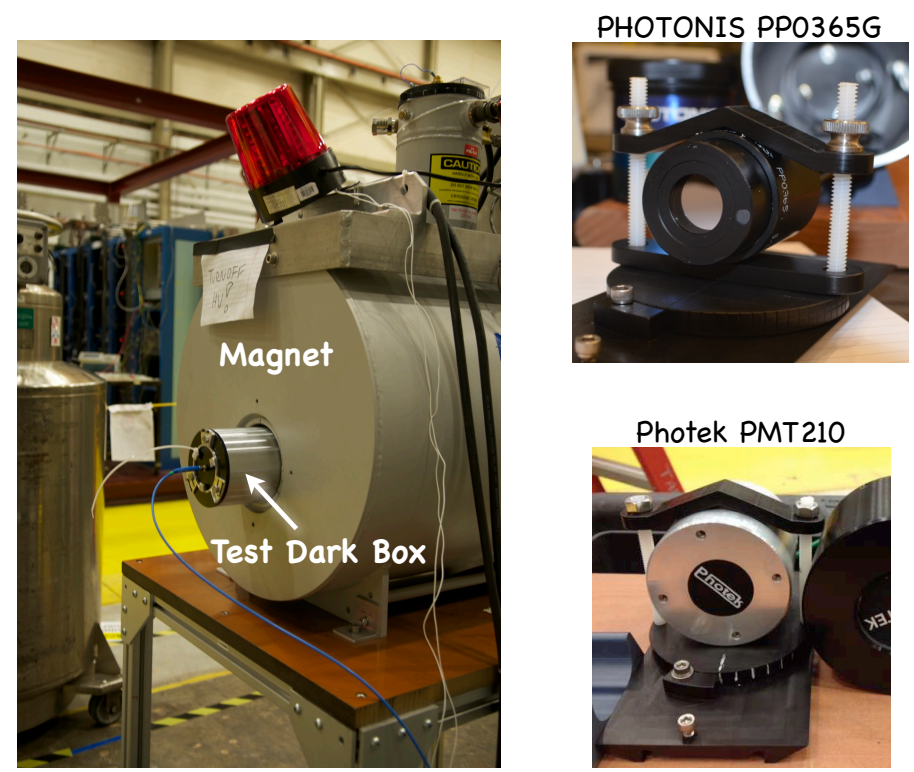

Figure 2. Left: A photograph of the dark box as it is placed in the bore of the superconducting magnet. Right: Photographs of the MCP PMTs being tested: PHOTONIS PP0365G (top) and Photek PMT210 (bottom). Each photomultiplier is kept in position by a custom-built holder.
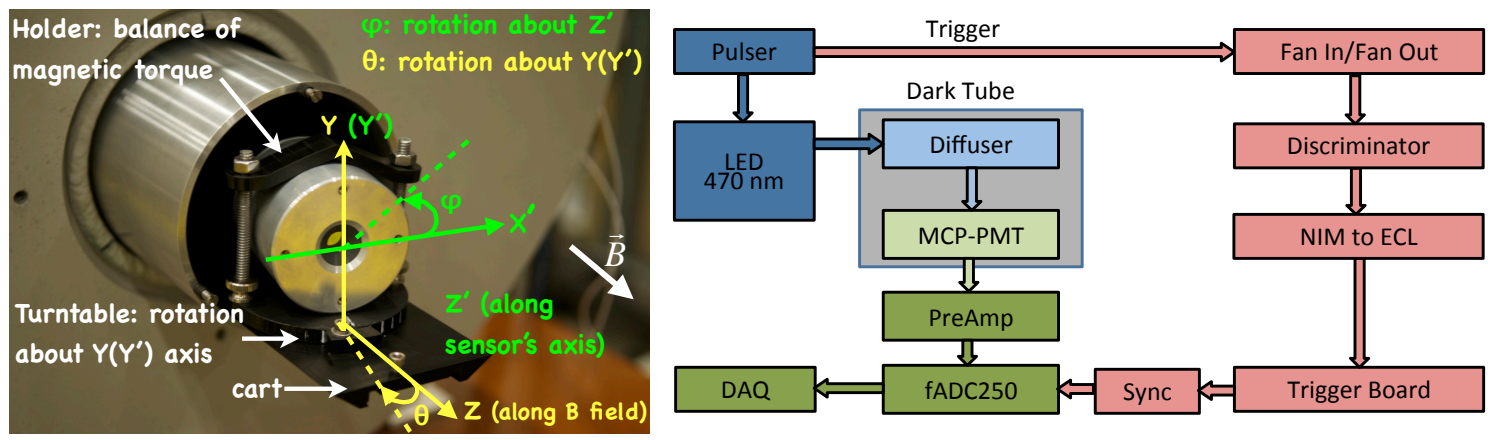

Figure 3. Left: A photograph of the dark box and the MCP-PMT setup that allows to hold the sensor in place and to rotate it around two axes, parallel ( $\mathrm{Z}^{\left(\mathrm{Z}^{\prime}\right)}$ axis) to and perpendicular $\left(\mathrm{Y}^{\left(\mathrm{Y}^{\prime}\right)}\right.$ axis) to the floor. The diameter and the length of the box are $11.5 \mathrm{~cm}$ and $46 \mathrm{~cm}$, respectively. The solenoidal magnetic field is parallel to the central axis of the dark box. Right: A flowchart of the MCP-PMT readout during our tests. Photons of wavelengths of $470 \mathrm{~nm}$ are incident on the photocathode and produce photoelectrons. A high voltage was applied between the photocathode and the anode in order to accelerate the photoelectrons and initiate an avalanche. The total charge was collected on the anode, amplified by a $200 \times$ preamplifier, and digitized by a flash analog-to-digital converter (fADC). The fADC was readout by a data acquisition system (DAQ).

on a turn table that allows for rotation around a vertical axis ( $\mathrm{Y}$ (Y') axis on left panel of Fig. 3). This rotation is described by a polar angle $\theta$. The accessible range of $\theta$ depends on the size of the sensor and is limited by the signal- and high-voltage cables installed on the back of the sensor. The MCP PMT is held in place by a holder and non-magnetic screws. A cart allows to move the sensor 
relative to the box for precise positioning and operation. The setup allows also for sensor rotation about an axis parallel to the floor $\left(Z\left(Z^{\prime}\right)\right.$ axis on the left panel in Fig. [1). This rotation is described by an azimuthal angle $\phi$.

The photocathode of the tested MCP PMT was illuminated by a pulser-driven ${ }^{1}$ light emitting diode (LED). The data shown here were taken with an LED of wavelength of $470 \mathrm{~nm}$. The light was transmitted to the dark box by means of an optical fiber and spread out by a diffuser installed on the inner side of the dark box's front cap. The diffused light has nearly constant intensity throughout the area of the photocathode. The pulser was operated in such a mode that the light incoming to the photocathode was at low intensity - the average number of photoelectrons was 0.15 and 1.2 for the $3-\mu \mathrm{m}$ and the $6-\mu \mathrm{m}$ sensors, respectively. The pulser was set to deliver 10-ns wide pulses with a frequency of $30 \mathrm{kHz}$. The output signal of the MCP PMT was amplified by a factor of $200^{2}$ and then input to a flash analog-to-digital converter (fADC) ${ }^{3}$. The latter was readout by a data acquisition system. The setup was triggered by the trigger output signals from the pulser. A flow chart of the MCP-PMT readout and signal processing is shown on the right diagram in Fig. 3 .

Two single-anode MCP PMTs were tested; PHOTONIS ${ }^{4}$ PP0365G (6- $\mu \mathrm{m}$ pore size, 18.2mm active diameter) and Photek ${ }^{5}$ PMT210 (3- $\mu \mathrm{m}$ pore size, $10-\mathrm{mm}$ active diameter), see right panel of Fig. 2. The response of each sensor was scanned at fixed $\theta$ angle from $0 \mathrm{~T}$ to $5 \mathrm{~T}$. For several $\theta$ angles, the B-field scans were done for various $\phi$ angles. In order to estimate systematic uncertainties due to instability of the pulser and reproducibility of the sensor location within the dark box, repeated measurements at some settings were randomly performed throughout the data taking.

\section{Results and Discussion}

The goal of our measurements is to evaluate the gain performance of MCP PMTs in magnetic fields up to $5 \mathrm{~T}$. As a control variable we use the average collected anode charge per pulse (pedestal appropriately subtracted), which should be proportional to the sensor's gain. To obtain this quantity, we integrate the signal waveform recorded by the fADC. The fADC samples the signal every $4 \mathrm{~ns}$ within a window of $1 \mu \mathrm{s}$. An example waveform for one event is shown on the left panel in Fig. 6 . In this paper we show results using integration over 9 bins. For each event $i$, the average pedestal was determined from the ADC digits in the first 20 bins. The waveform was then integrated over 9 bins, as shown in Fig. . and the integral of the pedestal over 9 bins was subtracted in order to obtain a value, $Q_{9, i}$, that is directly proportional to the total collected anode charge for that event. The quantity we report here for each setting $(\mathrm{B}, \theta, \phi)$, Average Charge (a.u.), is an average of the values $Q_{9, i}$ of all recorded events at that setting. Alternatively, one can integrate the pedestalsubtracted average waveform, which is shown on the right panel in Fig. $甘$. Both methods yield consistent results.

\footnotetext{
${ }^{1} \mathrm{HP} 8116 \mathrm{~A} 50 \mathrm{MHz}$ Programmable Pulse/Function Generator

${ }^{2}$ ORTEC VT120 Fast-Timing Preamplifier

${ }^{3}$ VME64x Flash ADC Module fADC250, Jefferson Lab Electronics Group

${ }^{4}$ PHOTONIS FRANCE S.A.S, Avenue Roger Roncier, 19100 Brive B.P. 520, 19106 BRIVE Cedex France. URL: http://www.photonis.com

${ }^{5}$ Photek, 26 Castleham Road, St. Leonards on Sea, East Sussex, TN38 9NS United Kingdom. URL: http://www.photek.com
} 

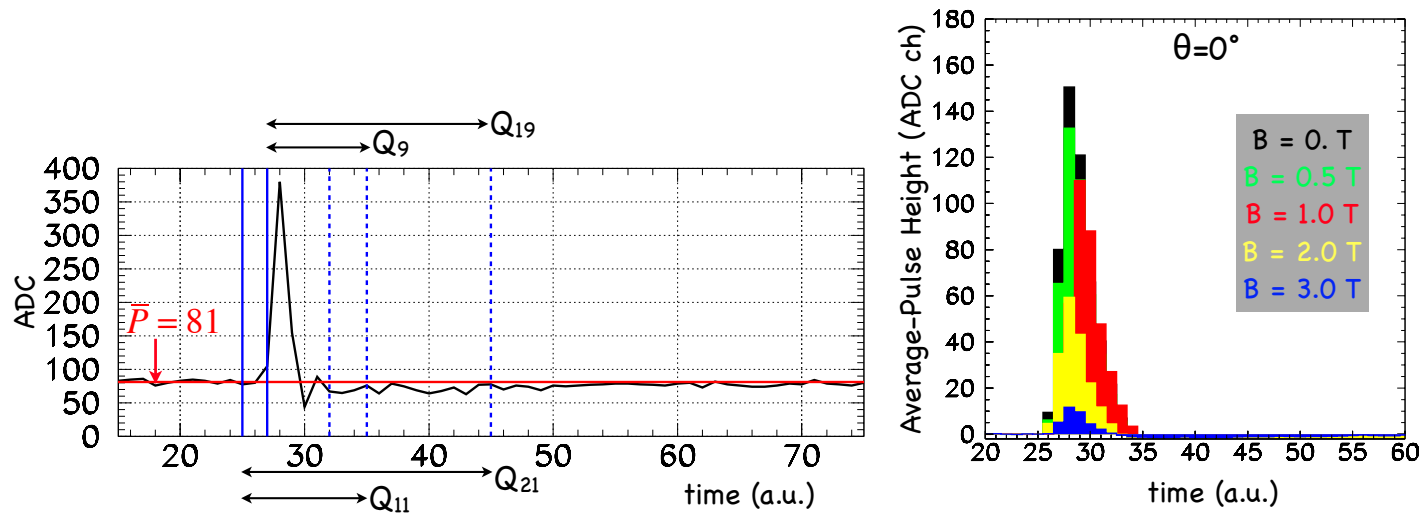

Figure 4. Left: An example waveform measured for PP0365G. The $X$ axis shows the time scale; one bin is 4-ns long. The $\mathrm{Y}$ axis shows the ADC digit. The fADC calibration is $19.1 \pm 0.2 \mathrm{fC} / \mathrm{ADC}$. The red solid line indicates the pedestal position for that event. The labels $\mathrm{Q}_{9}, \mathrm{Q}_{11}, \mathrm{Q}_{19}, \mathrm{Q}_{21}$ and the corresponding range indicators denote various integration ranges, over 9, 11, 19, and 21 bins, respectively, for the calculation of the total anode charge collected for that event. The limits and the width of the integration range were varied for systematic purposes. Right: Average waveforms obtained with PP0365G at $\theta=0^{\circ}$ and various B-field magnitudes. One clearly observes a decrease of the signal amplitude as the magnetic field magnitude increases.

Figure 5 shows the gain performance of the two tested sensors at nominal position, $\theta=0^{\circ}$ and $\phi=0^{\circ}$, for B-field magnitudes up to $5 \mathrm{~T}$. Data are shown for two values of the high voltage, $93 \%$ and $99 \%$ of the maximum manufacturer-recommended high-voltage value. For the $6-\mu$ m pore-size sensor, we observe a smoothly, nearly linearly, decreasing charge as the magnitude of the field increases. By increasing the high voltage to $99 \%$ of its maximum value, the operational range of the sensor can be increased up to $3.5 \mathrm{~T}$. For the $3-\mu \mathrm{m}$ pore-size sensor, we observe an increase of the anode charge up to $0.5 \mathrm{~T}$, followed by a smooth decrease as the magnitude of the field increases. By increasing the high voltage to $99 \%$ of its maximum value, the operational range of the sensor can be increased up to $5 \mathrm{~T}$. Overall, the data at $\theta=0^{\circ}$ suggest that the smaller pore-size sensor has better immunity to high magnetic fields as it can be operated up to higher fields and the collected anode charge decreases slower as the field magnitude increases. Also, at a given field, an increase of the high voltage seems to lead to a higher collected-charge increase. The preliminary uncertainty of 5\%, shown by the error bars of each point, is by far dominated by the systematic uncertainty. The latter was estimated as the standard deviation of the sample of repeated outcomes of the average anode charge at the same setting (notably $\theta=0^{\circ}, \phi=0^{\circ}$, and $\mathrm{B}=0 \mathrm{~T}$ ) from trials taken randomly throughout the measurement period. The standard deviation accounts for variations of the light intensity on the photocathode and of the positioning of the sensor within the dark box.

Figure 6 shows our results at other $\theta$ angles. The two sensors show very different responses. The B-field dependence of the average anode charge for the $6-\mu \mathrm{m}$ sensor exhibits a maximum below $1 \mathrm{~T}$ for $\theta=20^{\circ}, 25^{\circ}$, and $30^{\circ}$, whereas the dependences at $\theta=0^{\circ}$, and $10^{\circ}$ are smoothly decreasing as the B-field magnitude increases. As the B-field increases above $1 \mathrm{~T}$, the average collected charge decreases faster for the higher angles than for the lower angles.

A common characteristics of both sensors is that as the angle between the sensor and the field axes increases, the field range in which the sensor gives a reasonable response becomes narrower. 

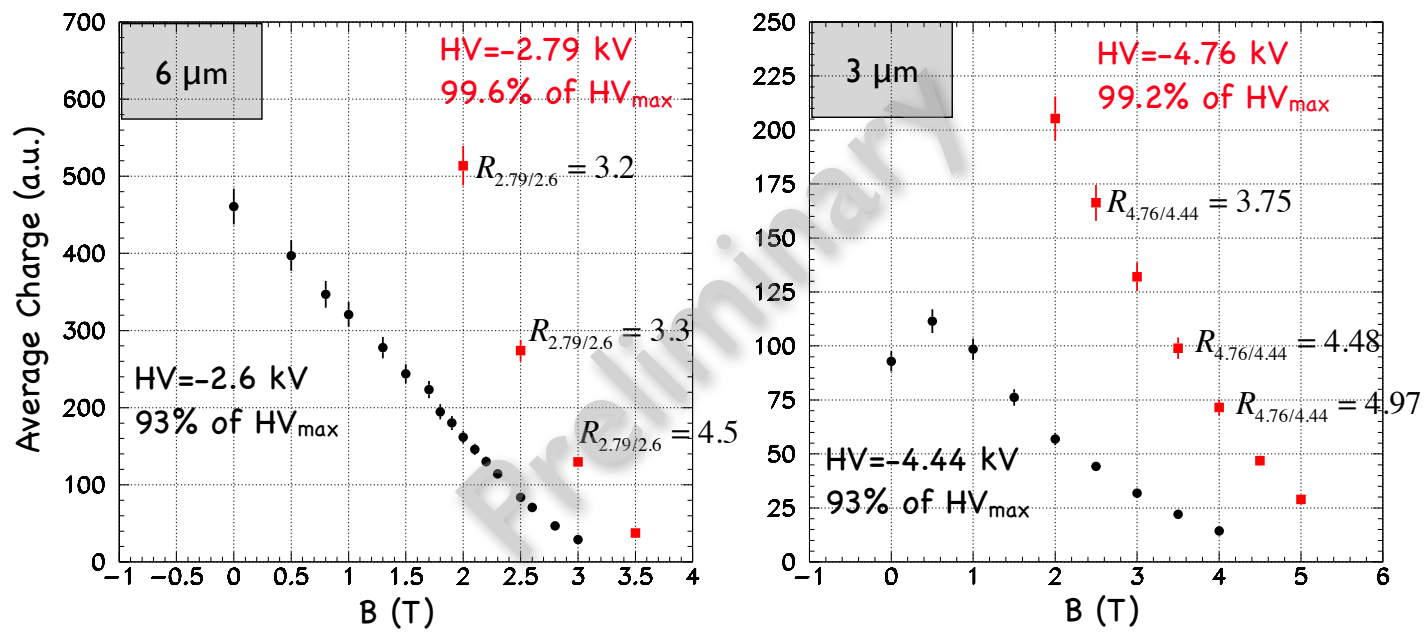

Figure 5. Left: Gain performance of PHOTONIS PP0365G at $\theta=0^{\circ}$ and two high-voltage values in magnetic field up to $3.5 \mathrm{~T}$. The solid black circles show the sensor response at a high voltage of $93 \%$ of the maximum high voltage. A reasonable signal can be obtained at fields up to $3 \mathrm{~T}$. The field range of operation can be increased by increasing the high voltage. The red solid squares show the sensor response at a high voltage of $99 \%$ of the maximum high voltage. A reasonable signal can be obtained at fields up to $3.5 \mathrm{~T}$. The total collected anode charge decreases by a factor of 15 as the field increases from $0 \mathrm{~T}$ to $3 \mathrm{~T}$. Right: Gain performance of Photek PMT210 at $\theta=0^{\circ}$ and two high-voltage values in magnetic field up to $5 \mathrm{~T}$. The solid black circles show the sensor response at a high voltage of $93 \%$ of the maximum high voltage. A reasonable signal can be obtained at fields up to $4 \mathrm{~T}$. The field range of operation can be increased to $5 \mathrm{~T}$ by increasing the high voltage to $99 \%$ of the maximum high voltage (red solid squares). The total collected anode charge decreases by a factor of 6 as the field increases from $0 \mathrm{~T}$ to $4 \mathrm{~T}$. The error bars of all the points include statistical and 5\% preliminary systematic uncertainties, with the latter by far dominating the former. The systematic uncertainty reflects variations in the reproducibility of the result for the same data point.

The difference between the $0^{\circ}$ and the larger-angle curves, however, is larger for the smaller poresize sensor, which at $0^{\circ}$ can be operated up to $5 \mathrm{~T}$, whereas at larger angles it can be operated only up to $2 \mathrm{~T}$. For a comparison, the $6-\mu \mathrm{m}$ pore-size sensor can be operated up to $3 \mathrm{~T}$ at $0^{\circ}$ and up to 2 $\mathrm{T}$ at larger angles. These data can also be presented as a function of angle for fixed field as shown in Fig. 7 . At all of the presented field magnitudes, the $3-\mu \mathrm{m}$ sensor data show a steep decrease from $0^{\circ}$ to $5^{\circ}$, above which the decrease continues at a smaller rate as a function of angle. The rate of decrease from $5^{\circ}$ to $20^{\circ}$ increases as the field magnitude increases, whereas above $20^{\circ}$ the slope of the angular dependences is very small and shows a weak dependence on the field magnitude. The $6-\mu \mathrm{m}$ sensor exhibits a maximum in the angular-dependence of the average collected charge at 0.5 $\mathrm{T}, 1 \mathrm{~T}$, and 1.5 T. The maximum seems to shift to lower angles as the field magnitude increases. At $2 \mathrm{~T}$, the total collected charge decreases smoothly as the $\theta$ angle increases. It should be noted that, while the data for the two sensors allow to make general conclusions about the effect of the pore size on the sensor performance, more detailed conclusions must be made with great caution as the orientation of the microchannel plates within the sensors are not necessarily the same. While we define the $\theta$ angle in the same fashion for both sensors, the orientation of the plates relative to the central axis of the sensor may strongly differ. We do not have details about the absolute orientation of the channels for either of the sensors. This information has not been necessary for 

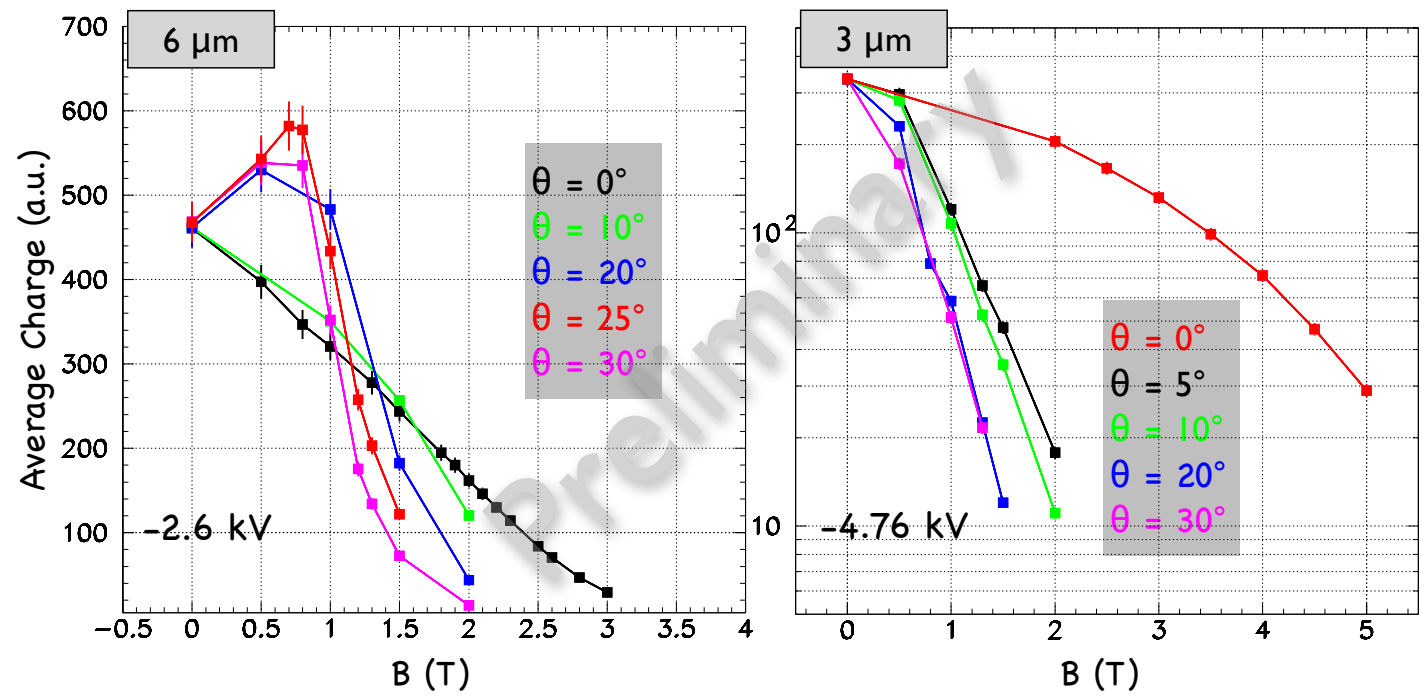

Figure 6. Left: Average collected anode charge as a function of B-field magnitude for $\theta$ angles up to $30^{\circ}$ for the $6-\mu \mathrm{m}$ pore-size sensor at a high voltage of $93 \%$ of the maximum value. Right: Average collected anode charge as a function of B-field magnitude for $\theta$ angles up to $30^{\circ}$ for the $3-\mu \mathrm{m}$ pore-size sensor at a high voltage of $99 \%$ of the maximum value.
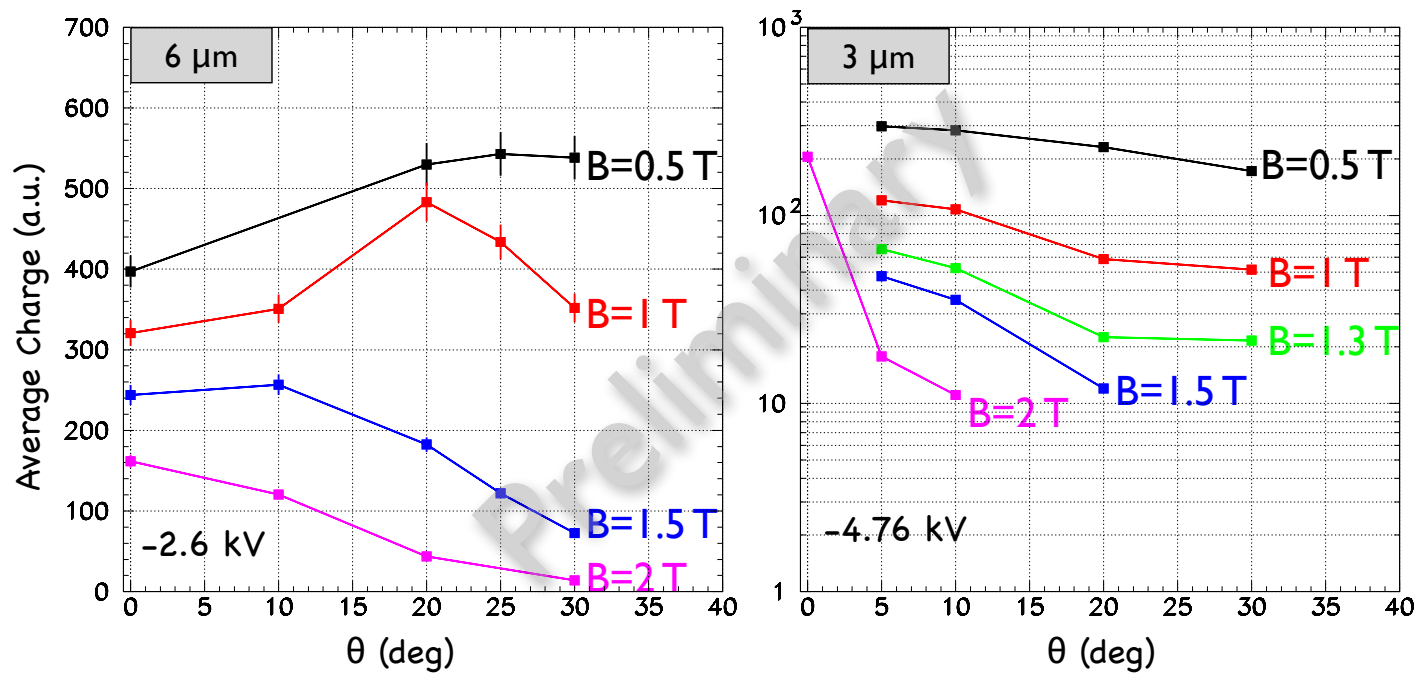

Figure 7. Same data as on previous figure but here presented as a function of the $\theta$ angle for fixed B-field magnitude.

current applications of MCP PMTs and so far has not been recorded in the manufacturing process of the sensor. Our data suggest that this will be important for the optimization of the sensor design and operational parameters for operations in areas of non-homogeneous high magnetic field, such as for DIRC in EIC.

Lastly, on Fig. 8 we show the effect of the $\phi$ angle on the sensor's response for various B-field magnitudes and $\theta$ angles. The data were taken with the 6- $\mu \mathrm{m}$ MCP PMT. Since the outer casing of the sensor is cylindrical and no specific orientation is marked as significant, we chose the $\phi=0^{\circ}$ position of the sensor randomly and marked it on the front side of the casing. We then measured 

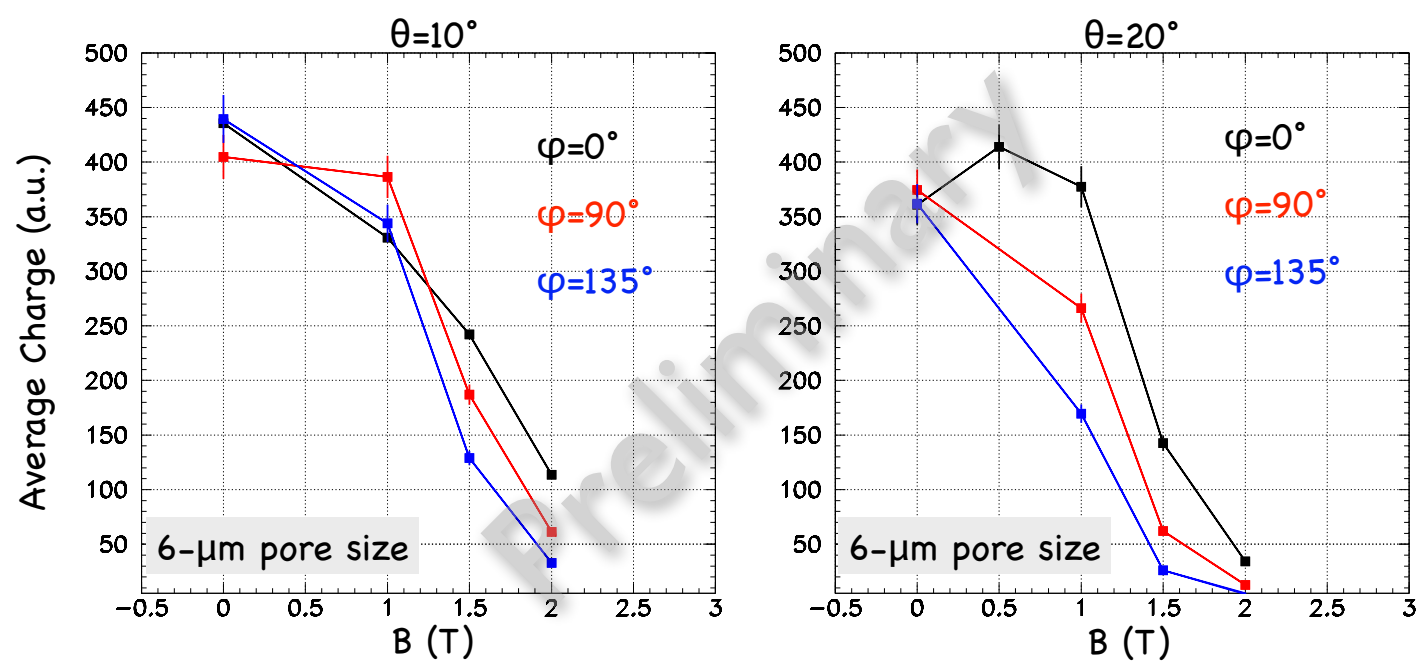

Figure 8. Average collected anode charge as a function of B-field magnitude for several $\phi$ angles and fixed $\theta$ angle of $10^{\circ}$ (left) and $20^{\circ}$ (right). The data were taken with the $6-\mu \mathrm{m}$ pore-size MCP PMT. The data show that at a given $\theta$ the collected charge as a function of field magnitude is $\phi$ dependent and that this dependence is different for different $\theta$.

the average collected anode charge as a function of field magnitude at $\phi=0^{\circ}, 90^{\circ}$, and $135^{\circ}$ for $\theta=10^{\circ}$ and $20^{\circ}$. The $\phi$ rotation was done counterclockwise about the sensor's axis when looked from the front. The data clearly show that at fixed $\theta$ the collected charge as a function of field magnitude is $\phi$ dependent and that the $\phi$ dependence is strongly correlated with the $\theta$ angle. The data at the larger $\theta$ angle show larger decreases of the collected charge as the $\phi$ angle increases.

\section{Summary}

We have mapped the gain performance of two single-anode MCP PMTs, PHOTONIS PP0365G (6- $\mu \mathrm{m}$ pore size) and Photek PMT210 (3- $\mu \mathrm{m}$ pore size) in magnetic field with magnitudes up to 5 $\mathrm{T}$ and various orientations of the sensor relative to the field direction. Overall, the smaller pore-size sensor showed a better magnetic-field immunity as it could be operated up to $5 \mathrm{~T}$. Our data suggest a very strong correlation between the gain performance in high magnetic field and the orientation of the sensor relative to the field. The shape of the dependence of the total collected anode charge on the field magnitude for various sensor orientations varies between the sensors, which may be partially due to the different orientation of the microchannel plates within the MCP PMT for the same $\theta$ and $\phi$ angles. Overall, the sensors gave a reasonable performance in fields up to 2 T. Our studies suggests that further design and operational parameter optimizations are needed in order to be able to operate the MCP PMTs with DIRC at EIC. To pursue this objective, we will extend our measurements to commercially available multi-anode MCP PMTs from various manufacturers and will develop an MCP-PMT simulation. The latter will allow to study the B-field performance of an MCP PMT as a function of design parameters that could not be easily varied in an experiment, such as bias angle, pore-size to channel-length ratio, distance between the microchannel plates, distance from the last plate to the anode, etc. The combination of real data and simulation studies will not 
only allow for design and operational optimization but also for establishing the performance limits of microchannel-plate photomultipliers as photon sensors in high magnetic fields.

\section{Acknowledgments}

The authors thank the Photek and the PHOTONIS companies for providing on loan MCP PMTs for the tests, the target group at Jefferson Lab for providing and operating the superconducting magnet, the detector group at Jefferson Lab for providing solutions and components for the mechanics in the dark box, and Sergey Boyarinov for his help with the data acquisition setup and operation. This work was supported in part by BNL under eRD4 and in part by the U.S. National Science Foundation under grant PHY-125782.

\section{References}

[1] A. Accardi et al., Electron Ion Collider: The Next QCD Frontier. Understanding the glue that binds us all, JLAB-PHY-12-1652, [arXiv:1212.1701].

[2] R. Tribble et al., The Frontiers of Nuclear Science. A Long Range Plan, NSAC Report 2007, http://science.energy.gov//media/np/nsac/pdf/docs/nuclear_science_low_res.pdf.

[3] D. Geesaman et al., Reaching for the Horizon. The 2015 Long Range Plan for Nuclear Science, NSAC Report 2015, http://science.energy.gov//media/np/nsac/pdf/2015LRP/2015_LRPNS_091815.pdf.

[4] G. Kalicy et al., DIRC detector for the future Electron Ion Collider experiment, Proceedings of the DIRC2015 Workshop, 11 - 13 November, Rauischholzhausen, Germany, see current issue of JINST.

[5] F. Uhlig et al., Performance studies of microchannel plate PMTs, Nucl. Instrum. Methods A 695 (2012) 68.

[6] A. Lehmann et al., Systematic studies of micro-channel plate PMTs, Nucl. Instrum. Methods A 639 (2011) 144 .

[7] A. Lehmann et al., Performance studies of microchannel plate PMTs in high magnetic fields, Nucl. Instrum. Methods A 595 (2008) 173.

[8] S. Hirose, Performance of the MCP-PMT for the Bell II TOP counter in a magnetic field, Nucl. Instrum. Methods A 639 (2011) 162.

[9] M. Akatsu et al., MCP-PMT timing property for single photons, Nucl. Instrum. Methods A 528 (2004) 763.

[10] S. Korpar et al., Photonis MCP PMT as a light sensor for the Belle II RICH, Nucl. Instrum. Methods A 766 (2014) 163.

[11] C.I. Coleman, Effects of perturbing magnetic fields on the performance of photoelectronic sensors, Rev. Sci. Instrum. 53, (1982) 53.

[12] G.W. Fraser, The gain, temporal resolution and magnetic-field immunity of microchannel plates, Nucl. Instrum. Methods A 291 (1990) 595.

[13] C.D. Keith et al., The Jefferson Lab frozen spin target, Nucl. Instrum. Methods A 684 (2012) 27.

[14] C.D. Keith et al., A polarized target for the CLAS detector, Nucl. Instrum. Methods A 501 (2003) 327. 